\title{
Intravesical BCG treatment causes a long-lasting reduction of recurrence and progression in patients with high-risk non-muscle-invasive bladder cancer
}

\author{
Tomas Thiel ${ }^{1,2,6}$ (D) Charlotta Ryk ${ }^{3} \cdot$ Lotta Renström-Koskela $^{1,2} \cdot$ Gunnar Steineck $^{4} \cdot$ Martin C. Schumacher $^{1,2}$. \\ N. Peter Wiklund ${ }^{1,2} \cdot$ Petra J. de Verdier $^{5}$
}

Received: 21 February 2018 / Accepted: 9 June 2018 / Published online: 15 June 2018

(c) The Author(s) 2018

\begin{abstract}
Purpose To analyse if BCG treatment leads to long-term reduction of recurrence, progression, and cancer-specific mortality (CSM) in patients with high-risk NMIBC.

Materials and methods 140 patients with high-risk NMIBC were drawn from a population-based cohort of 538 patients with newly diagnosed bladder cancer in the Stockholm County between 1995 and 1996. Data were collected prospectively, and a final follow-up for recurrence, progression, and CSM was performed after 15 years. Patients that received BCG were compared with patients who did not receive BCG. Survival analysis was done with Kaplan-Meier estimates and MantelCox log-rank test. Multivariable Cox proportional regression with stepwise selection was performed to verify the statistical significance of clinicopathological factors of prognostic importance. Results were displayed in Hazard ratios and a $p<0.05$ was considered to be statistically significant.

Results With a median follow-up of 100 months (2-182), 76 patients recurred; 50 progressed to muscle invasion; and 92 died of whom 38 died from bladder cancer. After 15-year follow-up, there was a statistically significant reduction in rate for recurrence (HR 0.40, $p<0.0001$ ) and progression (HR 0.52, $p=0.038$ ), but not for CSM, in patients that received BCG compared to those who did not.

Conclusions In this group, BCG in high-risk NMIBC patients reduced the long-term risk of recurrence and progression. The effect on CSM is yet to be clarified.
\end{abstract}

Keywords Non-muscle-invasive bladder cancer $\cdot$ BCG $\cdot$ Survival $\cdot$ Progression $\cdot$ Recurrence

Tomas Thiel

tomas.thiel@ki.se

Charlotta Ryk

lotta.ryk@gmail.com

Lotta Renström-Koskela

lotta.renstrom-koskela@sll.se

Gunnar Steineck

gunnar.steineck@oncology.gu.se

Martin C. Schumacher

m.schumacher@uro-hirslanden.ch

N. Peter Wiklund

peter.wiklund@sll.se

Petra J. de Verdier

Petra.deVerdier@ki.se
1 Department of Urology, Karolinska University Hospital, Stockholm, Sweden

2 Urology Laboratory, Department of Molecular Medicine and Surgery, Karolinska Institutet, Stockholm, Sweden

3 Department of Clintec, Karolinska Institutet, Stockholm, Sweden

4 Department of Oncology-Pathology, Karolinska Institutet, Stockholm, Sweden

5 Division of Clinical Chemistry, Department of Laboratory Medicine, Karolinska Institutet, Karolinska University Hospital, 14186 Stockholm, Sweden

6 Norra Kungsvägen 11b, 18131 Lidingö, Sweden 


\section{Introduction}

Intravesical Bacillus Calmette et Guerin (BCG) is an established treatment for non-muscle-invasive bladder cancer (NMIBC) and was introduced in 1976 by Morales [1]. The European association of Urology (EAU) recommends that all high-risk NMIBC patients should receive intravesical instillations of BCG [2]. According to a working group of EAU guidelines for NMIBC, high-risk NMIBC consists of all stage T1-, TaG3-, primary, and concomitant cancer in situ of the bladder (CIS) and recurrent and large TaG1G2 tumours [3]. EORTC developed risk tables to calculate a risk of recurrence and progression in an individual patient with NMIBC (1 and 5 years). Based on these probabilities, EAU working group suggested to stratify tumours in to three groups (low, intermediate, and high) to reflect the risk of both recurrence and progression using recurrence/progression score [4].

For high-risk NMIBC tumours, the risk of progression and cancer-specific mortality (CSM) is increased as compared to TaG1-G2 tumours [5]. Important factors associated with a high risk of recurrence are tumour size and number; important factors for progression and disease-specific survival are tumour grade and stage [2]. The 5-year progression rate for patients with $\mathrm{T} 1$ tumours ranges from 6 to $40 \%$ [4]. In patients with CIS, the corresponding rate is slightly higher (54\%) [6].

BCG is given as intravesical instillations [1] and the recommended treatment regimen includes an induction course of 6 weekly instillations followed by additional instillations for up to 36 months [3, 7]. While it is established that intravesical $\mathrm{BCG}$ reduces the number of recurrent NMIBC [8], there is an uncertainty whether BCG treatment prevents or delays progression to muscle invasion or metastases, or decreases CSM. While several studies report a decrease of progression and CSM after BCG treatment [9-11], a study by Malmström et al. from 2009 [8] suggests no such effect. Cochrane reports from 2003 and 2011 failed to see any effect of BCG on progression or cancer-specific mortality. Nevertheless, these reports indicate that BCG is superior to other intravesical agents, e.g., mitomycin and epirubicin, in reducing tumour recurrence $[12,13]$. It has been shown that BCG reduces recurrence in CIS patients [14, 15]. In BCG maintenance treatment for CIS, the complete response rate is as high as $84 \%$, although the 5-year disease free survival is lower $(70 \%)$ due to extravesical recurrence and progression [14].

The objective of this study was to analyse if BCG treatment reduces recurrence, progression, and CSM in patients with high-risk NMIBC. A 15-year clinical follow-up has been performed in a population-based study material consisting of the majority of patients with newly diagnosed high-risk NMIBC in the Stockholm county in 1995-1996 $(n=140)$.

\section{Materials and methods}

\section{Patient cohort and clinical assessment}

Over a period of 2 years, 1st of January 1995 to 31 st of December 1996, all patients newly diagnosed with urothelial carcinoma (UC) in the Stockholm County were asked to participate in a prospective cohort study. In total of 538 patients, $76 \%$ (538/705) of all cases in the county during that period, agreed to participate. The entire cohort was described in a 5-year clinical follow-up published in 2003 [5] and in an additional article in 2010 [16]. From this patient material, all cases with high-risk NMIBC were included in the present study, a total of 140 patients. The same pathologist performed all histopathological assessments and the standard pathological report included data on stage, grade, presence of concomitant CIS, and detrusor muscle in the specimen. The tumour, node, and metastasis (TNM) classification from 1978 was used for histopathological classification [17]. For tumour grading, the WHO 1999 malignancy grading system was applied [18]. High-risk NMIBC was defined as all non-muscle-invasive T1 and all G3 tumours, e.g., T1G1-G3, TaG3, and primary and secondary CIS, according to EAU standards [19] and previous results from our group [5].

The initial transurethral resection (TUR-BT) for staging and local control was performed in all patients. Treatment options for high-risk NMIBC were at that time TUR-BT only, or with adjuvant intravesical chemo- or immunotherapy. A second resection was not performed on a regular basis and only recommended when tumour size $>3 \mathrm{~cm}$. Data on residual tumours in this procedure were not registered systematically. Any additional treatment was offered to the discretion of the treating physician according to the local traditions and the patient's performance status and request, however. Immediate cystectomy was not standard and performed only in occasional cases $(n=3)$. Multidisciplinary conference was recommended but not performed for all newly diagnosed bladder cancers.

BCG immunotherapy (Connaught $2 \times 10^{8}$ to $3 \times 10^{9}$ bacteria/instillation) was given according to the manufacturer's recommendations as an induction course of 6 weekly instillations. Maintenance treatment, either monthly instillations or 3 weekly instillations every 3 months, (for at least 1 year), was recommended but not given according to a single standard. Data on the duration of BCG-treatment were not registered during the 15-year clinical follow-up and due to different archive systems in the participating hospitals it has not been possible to obtain this information retrospectively. Cystoscopy and cytology were included in 
the routine follow-up (every 3 months for the first year and every 6 months for the second year and thereafter annually for 3 years). A CT scan was performed only when there were symptoms of progression.

\section{Follow-up}

Patient records of all original participants were scrutinized in 2011. Follow-up time was defined as the time elapsed from date of diagnosis to death or last clinical evaluation. Parameters registered initially were as follows: date of diagnosis, sex, age, stage, grade, tumour size, multifocality, presence of concomitant CIS, and presence of detrusor muscle in the resected material. Parameters collected continuously and at the end of the study were: number and date of recurrence, date of progression to muscle-invasive tumour, development of lymph-node and distant metastasis, type of therapy, and date of and cause of death. EORTC score for risk of recurrence and progression was calculated, according to the methods defined by EORTC [2] based on the initial data, and categorized as of intermediate or high risk. In cases where cause of death was registered as bladder cancer, without any previous progression, date of progression was considered to be the same as date of death. All patients included in the 5-year clinical follow-up were also included in the follow-up after 15 years.

Diagnosis of any new tumour in the bladder after the initial staging and grading by TUR-BT was considered as recurrence. Growth of the initial tumour into the muscular layer of the bladder or beyond, local, and distant metastasis or death from bladder cancer was considered as progression. Cancer-specific death was defined as death from bladder cancer according to the Swedish cause of death register.

\section{Statistical analysis}

The present study was performed on a fixed prospective cohort. Patients who received BCG were compared with patients who did not, in terms of time to recurrence, progression, and CSM. Univariable analysis for each of these terms was done for Kaplan-Meier survival estimates and curves were compared using the Mantel-Cox log-rank test. Multivariable Cox proportional regression with stepwise selection was used to verify the prognostic significance of clinicopathological factors of importance (age, sex, stage, grade, tumour size, multiple tumours, and concomitant CIS). Results were expressed as hazard ratios and a $p<0.05$ was considered statistically significant. In many studies, primary CIS is considered to be a separate entity. In the present study, all primary CIS patients were given BCG, and separate analysis both with and without CIS were, therefore, carried out. Finally, as a sensitivity analysis, all patients with CIS (primary and concomitant) were excluded to examine the impact of BCG on papillary tumours alone, a condition in which BCG adjuvant treatment is secondary only to TURBT. Statistical analysis was performed with IBM ${ }^{\circledR}$ SPSS $^{\circledR}$ Statistics, version 21.0 software.

\section{Results}

\section{Cohort data}

In total, 140 patients were enrolled in the study. One patient was excluded due to inconsistent information on survival. Median age was 71.5 years and the ratio male:female was 2.2:1. Among the 139 patients' tumours, $78 \%$ were staged as $\mathrm{T} 1,14 \%$ as Ta and $8 \%$ as primary CIS. Predominant grade was G3 (60\%). Size $>3 \mathrm{~cm}$ was common (64\%), while multifocality was less frequent $(43 \%)$. According to EORTC scores for recurrence, most patients were at intermediate risk, while EORTC scores for progression indicated high risk for $>90 \%$ of the patients. Median follow-up was 100 months and none of the patients were lost after the first 5-year follow-up. BCG-treated patients were younger (68.5 vs. 72 years), were staged less as T1 (68 vs. 93\%), had more primary CIS (13 vs. $0 \%$ ) and concomitant CIS (34 vs. 5\%), and had more G3 tumours (71 vs. $46 \%$ ) than non-BCGtreated patients. Detrusor muscle was obtained in $83 \%$ of the patients with T1 tumours, and there was no significant difference between BCG-treated and non-BCG-treated patients (86 vs $83 \%$ ) (see Table 1 ).

The initial treatment in all cases was TUR-BT. Only 14 patients received a non-BCG adjuvant intravesical instillation. There were three cases of immediate cystectomy, while deferred cystectomy due to recurrence or progression was performed in 25 patients (18\%). Cystectomy was more common in non-BCG patients, while other treatment modalities were not (see Table 2).

Within 5 years of clinical follow-up, 29 patients (21\%) had died of urothelial carcinoma. Almost all patients who died of bladder cancer, 37 out of 38 patients, had died within 10 years from diagnosis. After 15 years of clinical followup, 92 patients (66\%) had died, of which 38 patients (27\%) died of urothelial carcinoma. Stage progression was seen in 50 patients (36\%) and recurrence in 76 patients (55\%) (see Table 3).

\section{Outcome from BCG treatment}

Patients that received BCG treatment had less recurrence than the group that did not receive BCG [hazard ratio (HR) $0.40, p<0.0001]$. In addition, after exclusion of patients with primary CIS tumours $(n=11)$, there was a statistically significant difference between the two groups (HR 0.40, $p<0.0001$ ) (see Table 4 and Fig. 1). 
Table 1 Baseline characteristics and follow-up of the 139 highrisk NMIBC patients

\begin{tabular}{|c|c|c|c|c|}
\hline Variable & BCG, $n(\%)$ & Non-BCG, $n(\%)$ & Total, $n(\%)$ & $p$ \\
\hline No. $(\%)$ & $82(59)$ & $57(41)$ & $139(100)$ & 0.002 \\
\hline Age, year (median) & 68.5 & 72 & 71.5 & 0.02 \\
\hline \multicolumn{5}{|l|}{ Sex } \\
\hline Male & $58(71)$ & $38(67)$ & $96(69)$ & \multirow[t]{2}{*}{0.61} \\
\hline Female & $24(29)$ & 19 (33) & $43(31)$ & \\
\hline \multicolumn{5}{|l|}{ Tumour stage } \\
\hline $\mathrm{Ta}$ & $15(18)$ & $4(7)$ & $19(14)$ & 0.06 \\
\hline $\mathrm{T} 1$ & $56(68)$ & $53(93)$ & $109(78)$ & 0.005 \\
\hline CIS & $11(13)$ & 0 & $11(8)$ & 0.004 \\
\hline \multicolumn{5}{|l|}{ Concomitant $\mathrm{CIS}^{\mathrm{a}}$} \\
\hline $\mathrm{Y}$ & $24(34)$ & $3(5)$ & $27(21)$ & \multirow[t]{2}{*}{0.0001} \\
\hline $\mathrm{N}$ & $47(66)$ & $54(95)$ & $101(79)$ & \\
\hline \multicolumn{5}{|l|}{ Grade } \\
\hline G1 & $3(4)$ & $3(5)$ & $6(4)$ & 0.69 \\
\hline G2 & $21(25)$ & $28(49)$ & $49(36)$ & 0.002 \\
\hline G3 & $58(71)$ & $26(46)$ & $84(60)$ & 0.003 \\
\hline \multicolumn{5}{|l|}{ No. tumours ${ }^{\mathrm{a}}$} \\
\hline 1 & $43(61)$ & $30(53)$ & $73(57)$ & \multirow[t]{2}{*}{0.37} \\
\hline$\geq 2$ & $28(39)$ & $27(47)$ & $55(43)$ & \\
\hline \multicolumn{5}{|l|}{$\operatorname{Size}^{\mathrm{a}}$} \\
\hline$<3 \mathrm{~cm}$ & $26(37)$ & $20(35)$ & $46(36)$ & \multirow[t]{2}{*}{0.85} \\
\hline$\geq 3 \mathrm{~cm}$ & $45(63)$ & $37(65)$ & $82(64)$ & \\
\hline \multicolumn{5}{|c|}{ Presence of detrusor (for stage T1 tumours) } \\
\hline $\mathrm{Y}$ & $48(86)$ & $44(83)$ & $91(83)$ & \multirow[t]{2}{*}{0.70} \\
\hline $\mathrm{N}$ & $8(14)$ & $9(17)$ & $18(17)$ & \\
\hline \multicolumn{5}{|l|}{ EORTC score progression $^{\mathrm{a}}$} \\
\hline Intermediate & $6(9)$ & $6(11)$ & $12(9)$ & \multirow[t]{2}{*}{0.69} \\
\hline High & $65(91)$ & $51(89)$ & $116(91)$ & \\
\hline \multicolumn{5}{|l|}{ EORTC score recurrence $^{\mathrm{a}}$} \\
\hline Intermediate & $63(89)$ & $53(93)$ & $116(91)$ & \multirow[t]{2}{*}{0.41} \\
\hline High & $8(11)$ & $4(7)$ & $12(9)$ & \\
\hline \multicolumn{5}{|l|}{ Follow-up } \\
\hline Range, months & $2-182$ & $4-182$ & $2-182$ & \\
\hline Median follow-up, months & 121 & 108 & 100 & \\
\hline Mean follow-up, months & 108 & 81 & 97 & 0.0001 \\
\hline
\end{tabular}

BCG Bacillus Calmette-Guérin, $C I S$ carcinoma in situ, EORTC The European Organization for research on treatment of cancer

${ }^{\text {aP }}$ rimary CIS not included
For progression, the results showed a statistically significant difference between the BCG-treated and the non-BCGtreated group, both before (HR 0.52, $p=0.038$ ) and after exclusion of patients with primary and concomitant CIS (HR $0.41, p=0.018$ ). However, after exclusion of primary CIS patients, significance was lost after adjustments for age and stage (HR $0.58 p=0.075$ ) (see Table 5 and Fig. 2).

Death from urothelial carcinoma occurred at a lower rate in the BCG-treated group as compared to the non-BCGtreated group [20 vs. 39\%, HR 0.40, $p=0.006$ (see Table 3)]. However, after adjustments for age, stage, tumour size, and number of tumours, no statistically significant difference could be measured (see Table 6 and Fig. 3.).

\section{Discussion}

In this study, we investigated the long-lasting effect of BCG in patients with high-risk NMIBC, on treatment outcome in terms of recurrence, progression, and CSM. There was a strong statistically significant difference in recurrence between patients that received BCG treatment and 
Table 2 Treatment other than BCG

\begin{tabular}{lllcl}
\hline & BCG, $n(\%)$ & Non-BCG, $n(\%)$ & Total, $n(\%)$ & $p$ \\
\hline Curative intention & & & & \\
Instillation (mitomycin) & $8(10)$ & $6(11)$ & $14(10)$ & 0.88 \\
Cystectomy & $10(12)$ & $15(27)$ & $25(18)$ & 0.03 \\
Immediate cystectomy & 0 & $3(5)$ & $3(2)$ & 0.04 \\
Radiotherapy & $3(4)$ & $3(5)$ & $6(4)$ & 0.65 \\
Chemotherapy & 0 & $3(5)$ & $3(2)$ & 0.04 \\
Adj/neoadj chemotherapy & $2(2)$ & $2(4)$ & $4(3)$ & 0.71 \\
Palliation & & & & \\
Salvage cystectomy & $1(1)$ & $1(2)$ & $11(8)$ & 0.79 \\
Radiotherapy & $3(4)$ & $8(14)$ & $2(1)$ & 0.02 \\
Chemotherapy & 0 & $2(4)$ & & 0.09 \\
\hline
\end{tabular}

Table 3 Clinical outcome $n(\%)$

\begin{tabular}{|c|c|c|c|c|}
\hline & BCG & Non-BCG & Total & $p$ \\
\hline \multicolumn{5}{|c|}{ Recurrence } \\
\hline Yes & $35(42.7)$ & $41(71.9)$ & $76(54.7)$ & \multirow[t]{2}{*}{0.001} \\
\hline No & $47(57.3)$ & $16(28.1)$ & $63(45.3)$ & \\
\hline \multicolumn{5}{|c|}{ Progression to $\mathrm{T} 2 \mathrm{NM}$} \\
\hline Yes & $21(25.6)$ & $29(50.9)$ & $50(36.0)$ & \multirow[t]{2}{*}{0.002} \\
\hline No & $61(74.4)$ & $28(48.1)$ & $89(64.0)$ & \\
\hline \multicolumn{5}{|l|}{ CSM } \\
\hline Yes & $16(19.5)$ & $22(39.0)$ & $38(27.3)$ & \multirow[t]{2}{*}{0.01} \\
\hline No & $66(80.5)$ & $35(61.0)$ & $101(72.7)$ & \\
\hline \multicolumn{5}{|c|}{ Overall death } \\
\hline Yes & $50(61.0)$ & $42(73.7)$ & $92(66.2)$ & \multirow[t]{2}{*}{0.42} \\
\hline No & $32(39.0)$ & $15(26.3)$ & 47 (33.8) & \\
\hline
\end{tabular}

$B C G$ Bacillus Calmette-Guérin, T2NM stage 2-4, lymph-node metastasis or distant metastasis or CSM, CSM cancer-specific mortality

Table 4 Univariable and multivariable analyses of time to recurrence

\begin{tabular}{|c|c|c|c|c|}
\hline & \multicolumn{2}{|l|}{ Univariable } & \multicolumn{2}{|l|}{ Multivariable } \\
\hline & HR $(95 \%$ CI $)$ & $p$ value & $\mathrm{HR}(95 \% \mathrm{CI})$ & $p$ value \\
\hline \multicolumn{5}{|l|}{ All patients } \\
\hline Non-BCG & 1.0 & & 1.0 & \\
\hline $\mathrm{BCG}$ & $\begin{array}{c}0.30(0.19- \\
0.48)\end{array}$ & $<0.0001$ & $\begin{array}{l}0.40(0.24- \\
0.65)\end{array}$ & $<0.0001$ \\
\hline \multicolumn{5}{|l|}{ Ex. CIS } \\
\hline Non-BCG & 1.0 & & 1.0 & \\
\hline BCG & $\begin{array}{c}0.34(0.21- \\
0.54)\end{array}$ & $<0.0001$ & $\begin{array}{c}0.40(0.24- \\
0.65)\end{array}$ & $<0.0001$ \\
\hline
\end{tabular}

Multivariable analysis after stepwise selection: age and concomitant CIS

$B C G$ Bacillus Calmette-Guérin, $C I$ confidence interval, $H R$ hazard ratio, $C I S$ cancer in situ the patients that did not, in favour of BCG treatment. In a study by Patard et al. [20], the recurrence rate was $50 \%$ for TUR-B + BCG vs $90 \%$ for TUR-B only. This result is similar to ours despite a longer follow-up in our study, probably reflecting observations from other studies [21] that most recurrence events in NMIBC patients occur within 5 years from primary diagnosis. In a retrospective study by Shahin et al. including only patients with T1G3 tumours, there was only a small difference in recurrence between the BCGtreated and the non-BCG-treated group (70 vs. 75\%) [22]. The patients in our study were more heterogenic in stage and grade and the difference in the estimated effect as compared to Shahin et al. most likely reflects T1 tumours being more aggressive than high-grade Ta tumours [2]. Thus, several studies have shown that $\mathrm{BCG}$ reduces recurrence in highrisk NMIBC, and the results in the present study confirm this notion.

Limitations when it comes to measure efficacy of BCG on tumour progression include heterogeneity of patient populations, a short follow-up period of most studies along with a nonuniform definition of disease progression [9, 21]. In the present study we had a well-defined group of patients (high-risk NMIBC), a long follow-up (15 years) and progression defined as an increase to stage $\mathrm{T} 2$ or higher or presence of lymph-node and distant metastases. With these criteria fulfilled, there was a statistically significant reduction of progression in the group of BCGtreated patients, which suggests that intravesical BCG has a long-lasting effect on progression. However, it has previously been suggested that BCG is less effective against T1G3 tumours with concomitant CIS [22], and in line with that, we did not find a significant association with BCG treatment and decrease of progression in this group. For patients with primary CIS, a comparison with the nonBCG-treated group was not possible in our study, since all primary CIS patients received BCG. However, in the 
Fig. 1 Recurrence-free survival according to treatment (BCG or not). $B C G$ Bacillus CalmetteGuérin

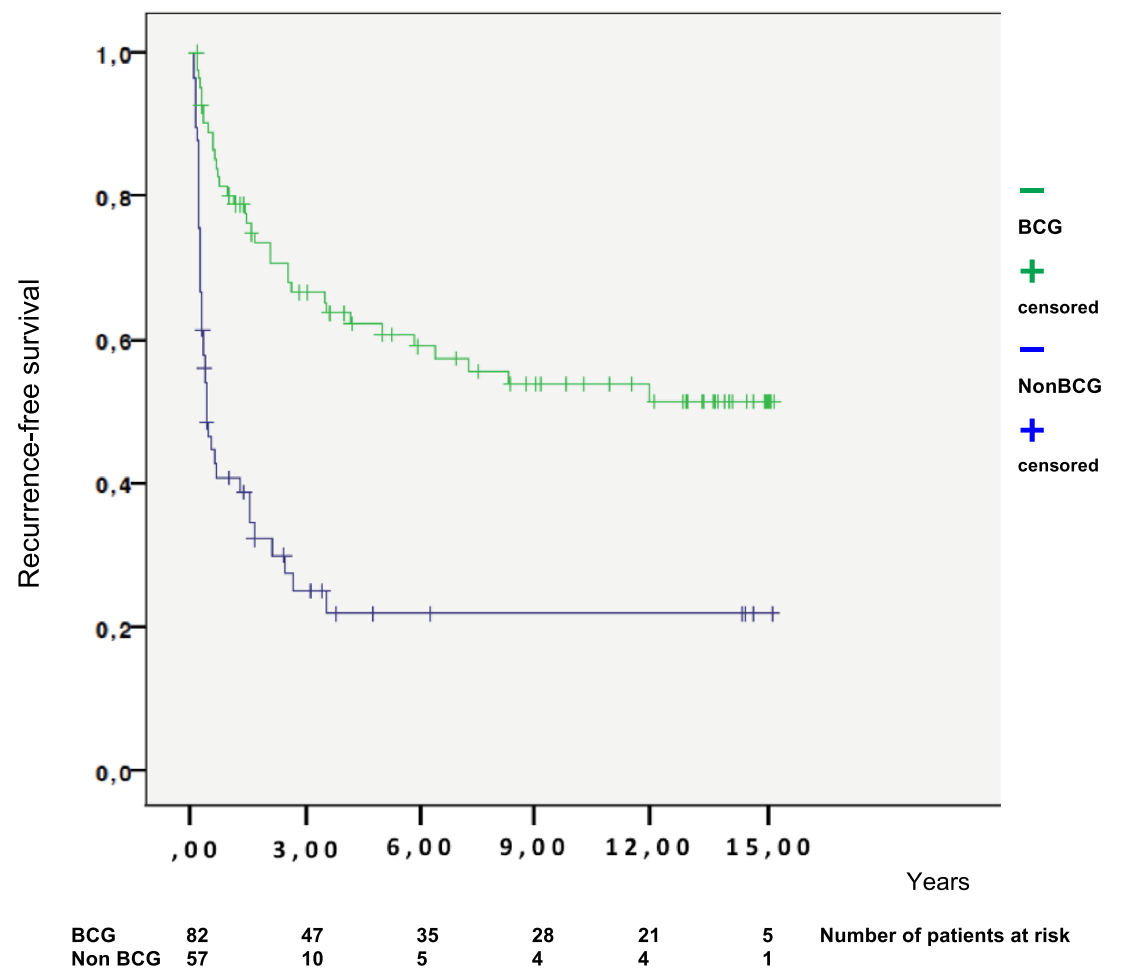

Table 5 Univariable and multivariable analyses of time to progression

\begin{tabular}{|c|c|c|c|c|}
\hline & \multicolumn{2}{|l|}{ Univariable } & \multicolumn{2}{|l|}{ Multivariable } \\
\hline & $\operatorname{HR}(95 \% \mathrm{CI})$ & $p$ value & $\operatorname{HR}(95 \% \mathrm{CI})$ & $p$ value \\
\hline \multicolumn{5}{|l|}{ All patients } \\
\hline Non-BCG & 1.0 & & 1.0 & \\
\hline BCG & $\begin{array}{c}0.38(0.22- \\
0.67)\end{array}$ & 0.001 & $\begin{array}{l}0.52(0.28- \\
0.97)\end{array}$ & 0.038 \\
\hline \multicolumn{5}{|l|}{ Ex. CIS } \\
\hline Non-BCG & 1.0 & & 1.0 & \\
\hline $\mathrm{BCG}$ & $\begin{array}{l}0.41(0.23- \\
0.72)\end{array}$ & 0.002 & $\begin{array}{l}0.58(0.32- \\
1.06)\end{array}$ & NS $(0.075)$ \\
\hline \multicolumn{5}{|c|}{ Ex CIS and concomitant CIS } \\
\hline Non-BCG & 1.0 & & 1.0 & \\
\hline BCG & $\begin{array}{c}0.30(0.15- \\
0.61)\end{array}$ & 0.001 & $\begin{array}{c}0.41(0.20- \\
0.86)\end{array}$ & 0.018 \\
\hline
\end{tabular}

Multivariable analysis after stepwise selection: age, concomitant CIS, and stage

$B C G$ Bacillus Calmette-Guérin, $C I$ confidence interval, $H R$ hazard ratio, $C I S$ cancer in situ, $N S$ non-significant

present study, only 1 out of $11(9 \%)$ patients with primary CIS progressed. This result contrasts with the previous observations that untreated CIS has a progression rate of over 50\% [14], and suggests a positive effect of BCGtreatment. Another observation in the present study was the association between BCG treatment and a decrease in progression in patients with high-risk papillary tumours without concomitant CIS. Previously published studies by Patard et al. [20] and Sylvester et al. [11] have shown similar effects.

CSM in our study affected $27 \%$ of the cohort. CSM in the BCG-treated group of patients was almost half that seen in the non-BCG-treated group. $80 \%$ of the deaths occurred within 5 years from diagnosis and only one death occurred later than 10 years from diagnosis. Overall mortality was 66\% during the 15-year follow-up. Despite a well-defined patient cohort in a prospective study with a long follow-up and a high death rate as indicated above, there was no statistically significant difference between the two treatment groups following multivariable analysis when primary CIS was excluded. This finding corroborate with the fact that no randomized study has shown a reduction in cancer-specific mortality in patients with high-risk NMIBC after the initial treatment with BCG. One explanation might be the influence of competing mortality as indicated by the high rate of overall mortality in the present study and the small sample size of only 140 patients.

In our study, $36 \%$ of the patients suffered from progression. In the group of patients who were given intravesical BCG, progression rate was lower $(26 \%)$, but still the rates are comparatively high. In a multicenter study of NMIBC by Cambier et al. [23] and in retrospective studies of highrisk NMIBC by Baniel et al. [24] and Hurle et al. [25], the progression rates were 20,8 , and $18 \%$ respectively. In these studies, however, the median follow-up was shorter (60, 56 and 85 months) as compared to the present study 
Fig. 2 Progression-free survival according to treatment (BCG or not). $B C G$ Bacillus CalmetteGuérin

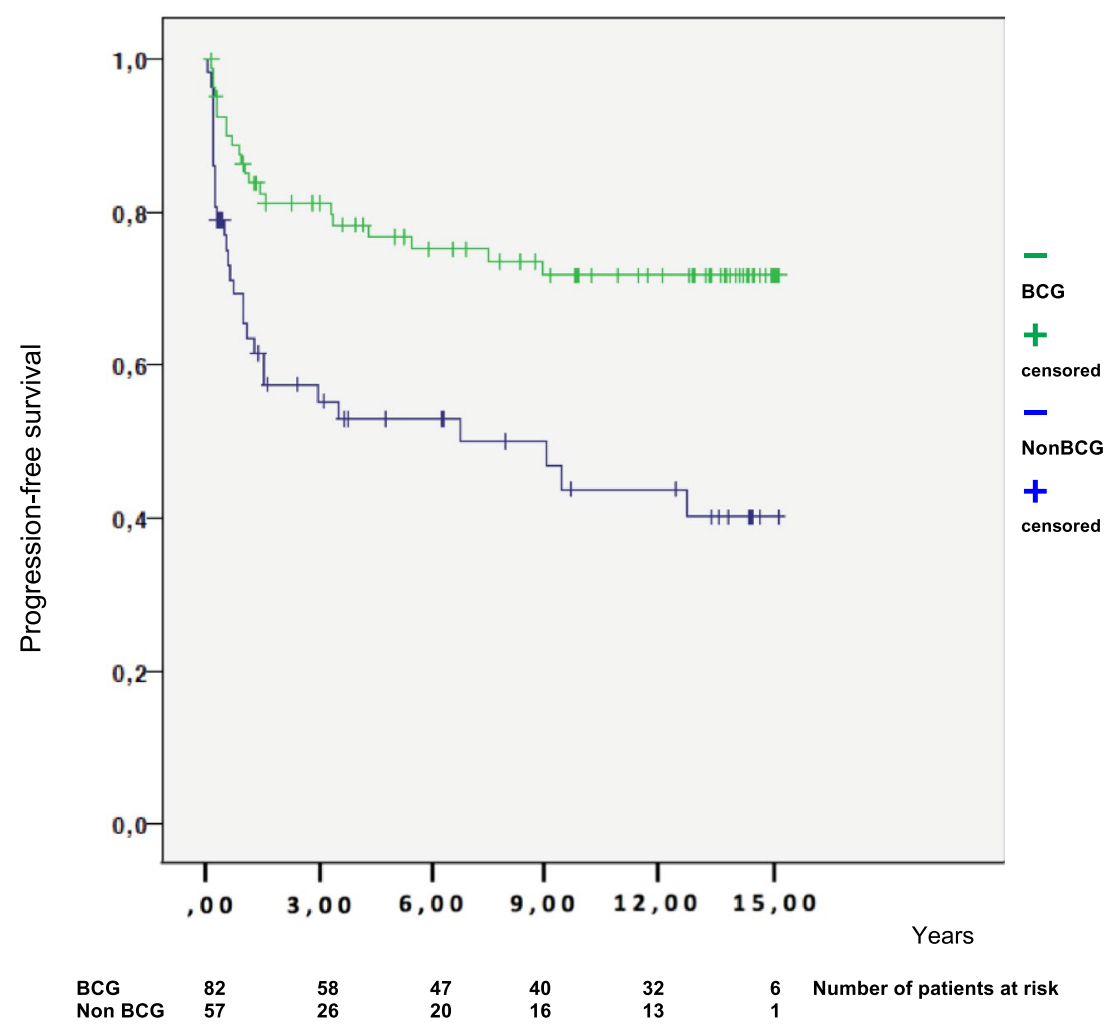

Table 6 Univariable and multivariable analyses of time to CSM

\begin{tabular}{|c|c|c|c|c|}
\hline & \multicolumn{2}{|l|}{ Univariable } & \multicolumn{2}{|l|}{ Multivariable } \\
\hline & HR $(95 \%$ CI $)$ & $p$ value & $\mathrm{HR}(95 \% \mathrm{CI})$ & $p$ value \\
\hline \multicolumn{5}{|l|}{ All patients } \\
\hline Non-BCG & 1.0 & & 1.0 & \\
\hline BCG & $0.40(0.21-0.77)$ & 0.006 & $0.58(0.29-1.16)$ & NS \\
\hline \multicolumn{5}{|l|}{ Ex. CIS } \\
\hline Non-BCG & 1.0 & & 1.0 & \\
\hline BCG & $0.45(0.24-0.86)$ & 0.016 & $0.78(0.39-1.55)$ & NS \\
\hline \multicolumn{5}{|c|}{ Ex CIS and concomitant CIS } \\
\hline Non-BCG & 1.0 & & 1.0 & \\
\hline BCG & $0.46(0.22-0.94)$ & 0.034 & $0.75(0.35-1.62)$ & NS \\
\hline
\end{tabular}

Multivariable analysis after stepwise selection: age and stage

$B C G$ Bacillus Calmette-Guérin, $C I$ confidence interval, CSM cancerspecific mortality, $H R$ hazard ratio, $C I S$ cancer in situ, $N S$ non-significant

(121 months) and only BCG-treated patients with T1G3 tumours were included.

Detrusor muscle presence in first TUR-BT is considered to be a surrogate marker of resection quality that predicts risk of the early recurrence [26]. In our study, detrusor muscle was present in $83 \%$ of the $\mathrm{T} 1$ tumours, indicating a high resection quality in Stockholm county in 1995 and 1996. However, there was no significant difference between BCG-treated patients and non-BCG-treated patients in the presence of detrusor muscle that would explain any difference of outcome between the two groups.

Our study was a prospective cohort study with a non-randomized inclusion of almost all high-risk NMIBC patients within a specified residence area during 24 months. Although the patient group that received BCG differed slightly, in terms of higher degrees of high-grade and Ta and CIS tumours, from the patient group that did not receive BCG, the EORTC scores for risk of recurrence and progression were similar between the two groups at base line. To compensate for the differences between the two groups, we performed multivariable analysis, but there might still be confounders (e.g., smoking) that we have not been able to compensate for. In addition, several previous studies $[8,21]$ have shown that maintenance treatment increases the effect of BCG on recurrence and progression. Unfortunately, from the clinical data, for this study, we were not able to obtain information about length and frequency of BCG treatment. BCG treatment was recommended for these patients, and when there was a decision not to use BCG, this was based on local traditions and the request and performance status of every patient. Particular reasons for not giving BCG treatment were not registered, however. 
Fig. 3 CSM-free survival according to treatment (BCG or not). $C S M$ cancer-specific mortality, $B C G$ Bacillus Calmette-Guérin

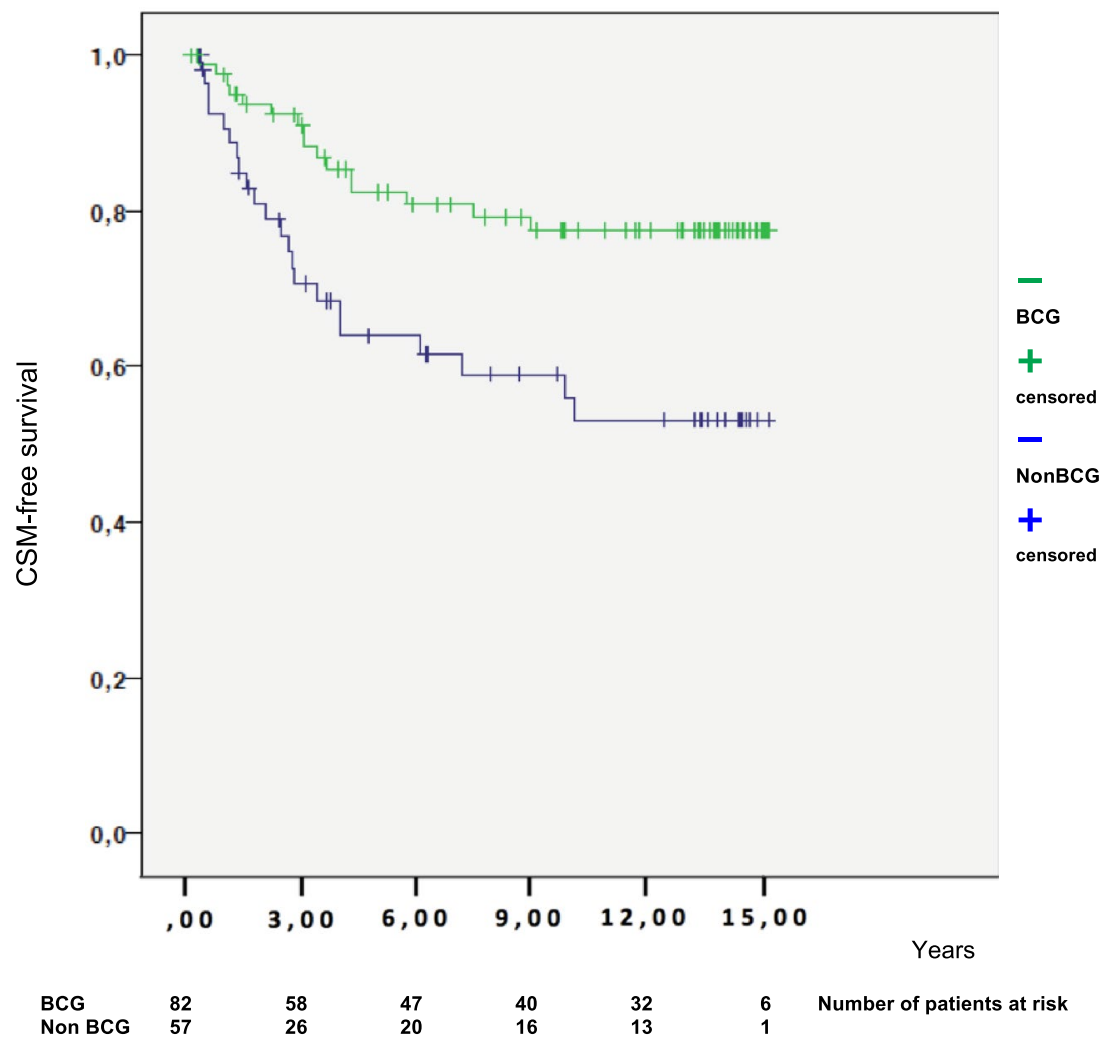

\section{Conclusion}

The present study supports the previous findings that intravesical treatment with $\mathrm{BCG}$ in patients with high-grade NMIBC decreases recurrence, regardless of maintenance treatment and a number of risk factors, e.g., grade and stage. We also show that BCG reduces progression in papillary tumours without concomitant CIS. The effect on recurrence and progression was long-lasting and the results of our study support the established recommendation of using BCG as the first-line adjuvant treatment in patients with high-risk NMIBC, especially in patients with primary CIS or papillary tumours without concomitant CIS. The question of whether $\mathrm{BCG}$ reduces CSM or not remains to be solved.

Acknowledgements Financial support was provided by the Cancer Society in Stockholm

Author contributions TT project development, data collection and management, data analysis, and manuscript writing. CR data analysis and manuscript editing. LR-K project development and data collection. GS protocol development, data collection, and management. MS data collection and management. NPW project development. PJV project development, data analysis, and manuscript editing.

\section{Compliance with ethical standards}

Conflict of interest There were no potential conflicts of interests among the authors of this article.
Human participants Research involving human participants was supervised and approved by the Regional Ethics board of the Stockholm County.

Informed consent All the participants in this study gave informed consent.

Open Access This article is distributed under the terms of the Creative Commons Attribution 4.0 International License (http://creativeco mmons.org/licenses/by/4.0/), which permits unrestricted use, distribution, and reproduction in any medium, provided you give appropriate credit to the original author(s) and the source, provide a link to the Creative Commons license, and indicate if changes were made.

\section{References}

1. Morales A, Eidinger D, Bruce AW (1976) Intracavitary Bacillus Calmette-Guerin in the treatment of superficial bladder tumours. J Urol 116(2):180-183

2. Babjuk M, Oosterlinck W, Sylvester R et al (2011) EAU guidelines on non-muscle-invasive urothelial carcinoma of the bladder, the 2011 update. Eur Urol 59(6):997-1008

3. Babjuk M, Burger M, Zigeuner R et al (2013) EAU guidelines on non-muscle-invasive urothelial carcinoma of the bladder: update 2013. Eur Urol 64(4):639-653

4. Sylvester RJ, van der Meijden AP, Oosterlinck W et al (2006) Predicting recurrence and progression in individual patients with stage Ta T1 bladder cancer using EORTC risk tables: a combined analysis of 2596 patients from seven EORTC trials. Eur Urol 49(3):466-477 (discussion 475-467) 
5. Larsson $P$, Wijkstrom $H$, Thorstenson A et al (2003) A populationbased study of 538 patients with newly detected urinary bladder neoplasms followed during 5 years. Scand J Urol Nephrol 37(3):195-201

6. Hudson MA, Herr HW (1995) Carcinoma in situ of the bladder. J Urol. 153(3 Pt 1):564-572

7. Kamat AM, Lamm DL (2000) Intravesical therapy for bladder cancer. Urology. 55(2):161-168

8. Malmstrom PU, Sylvester RJ, Crawford DE et al (2009) An individual patient data meta-analysis of the long-term outcome of randomised studies comparing intravesical mitomycin $\mathrm{C}$ versus bacillus Calmette-Guerin for non-muscle-invasive bladder cancer. Eur Urol 56(2):247-256

9. Bohle A, Bock PR (2004) Intravesical bacille Calmette-Guerin versus mitomycin $\mathrm{C}$ in superficial bladder cancer: formal metaanalysis of comparative studies on tumour progression. Urology 63(4):682-686 (discussion 686-687)

10. Millan-Rodriguez F, Chechile-Toniolo G, Salvador-Bayarri J, Palou J, Vicente-Rodriguez J (2000) Multivariate analysis of the prognostic factors of primary superficial bladder cancer. J Urol 163(1):73-78

11. Sylvester RJ, van der MA, Lamm DL (2002) Intravesical bacillus Calmette-Guerin reduces the risk of progression in patients with superficial bladder cancer: a meta-analysis of the published results of randomized clinical trials. J Urol 168(5):1964-1970

12. Shang PF, Kwong J, Wang ZP et al (2011) Intravesical Bacillus Calmette-Guerin versus epirubicin for Ta and T1 bladder cancer. Cochrane Database Syst Rev (5):CD006885

13. Shelley MD, Court JB, Kynaston H, Wilt TJ, Coles B, Mason M (2003) Intravesical bacillus Calmette-Guerin versus mitomycin $\mathrm{C}$ for Ta and T1 bladder cancer. Cochrane Database Syst Rev (3):CD003231

14. Lamm DL (1992) Carcinoma in situ. Urol Clin $\mathrm{N}$ Am 19(3):499-508

15. Kaasinen E, Wijkstrom H, Rintala E, Mestad O, Jahnson S, Malmstrom PU (2016) Seventeen-year follow-up of the prospective randomized Nordic CIS study: BCG monotherapy versus alternating therapy with mitomycin $\mathrm{C}$ and $\mathrm{BCG}$ in patients with carcinoma in situ of the urinary bladder. Scand J Urol 50(5):360-368

16. Thorstenson A, Schumacher MC, Wiklund NP et al (2010) Diagnostic random bladder biopsies: reflections from a populationbased cohort of 538 patients. Scand J Urol Nephrol 44(1):11-19
17. International Union against Cancer, Harmer MH, International Union against Cancer (1978) Committee on TNM Classification. TNM classification of malignant tumours, 3rd edn. The Union, Geneva

18. Reuter VE, Epstein JI, Amin MB, Mostofi FK (1999) The "WHO/ ISUP consensus classification of urothelial (transitional cell) neoplasms": continued discussion. Hum Pathol 30(7):879-880

19. Persad R, Lamm D, Brausi M et al (2008) Current approaches to the management of non-muscle invasive bladder cancer: comparison of current guidelines and recommendations. Eur Urol Suppl 7(10):637-650

20. Patard JJ, Rodriguez A, Leray E, Rioux-Leclercq N, Guille F, Lobel B (2002) Intravesical Bacillus Calmette-Guerin treatment improves patient survival in T1G3 bladder tumours. Eur Urol 41(6):635-641 (discussion 642)

21. Gontero P, Sylvester R, Pisano F et al (2015) Prognostic factors and risk groups in T1G3 non-muscle-invasive bladder cancer patients initially treated with Bacillus Calmette-Guerin: results of a retrospective multicenter study of 2451 patients. Eur Urol 67(1):74-82

22. Shahin O, Thalmann GN, Rentsch C, Mazzucchelli L, Studer UE (2003) A retrospective analysis of 153 patients treated with or without intravesical bacillus Calmette-Guerin for primary stage T1 grade 3 bladder cancer: recurrence, progression and survival. J Urol 169(1):96-100 (discussion 100)

23. Cambier S, Sylvester RJ, Collette L et al (2016) EORTC nomograms and risk groups for predicting recurrence, progression, and disease-specific and overall survival in non-muscle-invasive stage Ta-T1 urothelial bladder cancer patients treated with 1-3 years of maintenance Bacillus Calmette-Guerin. Eur Urol 69(1):60-69

24. Baniel J, Grauss D, Engelstein D, Sella A (1998) Intravesical bacillus Calmette-Guerin treatment for Stage T1 grade 3 transitional cell carcinoma of the bladder. Urology 52(5):785-789

25. Hurle R, Losa A, Manzetti A, Lembo A (1999) Intravesical bacille Calmette-Guerin in Stage T1 grade 3 bladder cancer therapy: a 7-year follow-up. Urology 54(2):258-263

26. Mariappan P, Zachou A, Grigor KM, Edinburgh Uro-Oncology G (2010) Detrusor muscle in the first, apparently complete transurethral resection of bladder tumour specimen is a surrogate marker of resection quality, predicts risk of early recurrence, and is dependent on operator experience. Eur Urol 57(5):843-849 